\title{
Memory Awareness Influences Everyday Decision Making Capacity about Medication Management in Alzheimer's Disease
}

\author{
Stephanie Cosentino, ${ }^{1}$ Janet Metcalfe, ${ }^{2}$ Mark S. Cary, ${ }^{3}$ Jessica De Leon, ${ }^{1}$ and Jason Karlawish ${ }^{4}$ \\ ${ }^{1}$ Cognitive Neuroscience Section of the Gertrude H. Sergievsky Center, Taub Institute for Research on Alzheimer's \\ Disease and the Aging Brain, and the Department of Neurology, Columbia University Medical Center, 630 West 168th Street, \\ P\&S Mailbox 16, New York, NY 10032, USA \\ ${ }^{2}$ Department of Psychology, Columbia University, New York, NY 10027, USA \\ ${ }^{3}$ Center for Clinical Epidemiology and Biostatistics, University of Pennsylvania, Philadelphia, PA 19104, USA \\ ${ }^{4}$ Departments of Medicine and Medical Ethics, Alzheimer's Disease Center, Institute on Aging, Center for Health Incentives, \\ University of Pennsylvania, Philadelphia, PA 19104, USA
}

Correspondence should be addressed to Stephanie Cosentino, sc2460@columbia.edu

Received 15 December 2010; Revised 15 March 2011; Accepted 28 March 2011

Academic Editor: Scott Kim

Copyright () 2011 Stephanie Cosentino et al. This is an open access article distributed under the Creative Commons Attribution License, which permits unrestricted use, distribution, and reproduction in any medium, provided the original work is properly cited.

\begin{abstract}
Memory awareness in early Alzheimer's disease (AD) influences capacity to provide informed consent for a memory treatment. This study investigated the extent to which aspects of memory awareness influence everyday decision-making capacity about medication management in AD. 42 participants with mild $\mathrm{AD}$ and 50 healthy elders underwent clinical ratings of memory awareness, metamemory testing, and an interview of everyday decision-making capacity regarding medication management. $45 \%$ of $\mathrm{AD}$ subjects were classified as aware (AAD) and 55\% as unaware (UAD) based on clinical ratings and supported by metamemory testing $(P=.015)$. Capacity was impaired in each of the $\mathrm{AD}$ groups as compared to the healthy elders $F(2,67)=17.63$, UAD, $P<.01$ AAD, $P=.01)$. Within the AD group, capacity correlated selectively with awareness as measured with clinical ratings $(r=-.41, P=.007)$ but not objective metamemory testing $(r=-.10, P=.60)$. Appreciation scores were lower in UAD as compared with $\operatorname{AAD} F(1,35)=8.36, P=.007$. Unawareness of memory loss should heighten clinicians' concern about everyday decision-making capacity in $\mathrm{AD}$.
\end{abstract}

\section{Introduction}

Decision-making capacity is fundamental to an individual's independence and invariably deteriorates at some point along the dementia continuum, compromising autonomy in financial matters [1], medical care $[2,3]$, and informed consent [4]. Even at the earliest stages of Alzheimer's disease $(\mathrm{AD})$, decision making can be affected, and certain features of the disease may have direct implications for decision making capacity in everyday life.

Previous work has demonstrated an important role for metacognitive factors including memory awareness and disease awareness in determining the likelihood that an individual would demonstrate capacity to make a decision about a treatment for $\mathrm{AD}$ [5]. While global cognition also influenced capacity in this study, there was not a direct relationship between these variables such that individuals at any given level of cognition could have been judged as competent or not competent. Consideration of the elements that constitute capacity reveals why memory awareness may have clear relevance for decisions about a memory treatment. Decision-making capacity has been conceptualized in terms of four core components including (1) understanding = The ability to comprehend information relevant to the decision (e.g., risks and benefits), (2) appreciation $=$ The ability to apply the information to one's own situation, (3) reasoning $=$ The ability to consider and compare potential consequences of various decisions, and (4) expression of choice $=$ the ability to communicate a stable choice $[6,7]$. If an individual is unaware that his or her memory is impaired, then he or she may be unlikely to appreciate the personal benefits of a proposed memory treatment, or to accurately 
anticipate and reason through the personal consequences of a decision. In fact, reasoning and appreciation of benefit were the decision-making indices most related to disordered awareness in the above study [5].

The integral role for self awareness in treatment decisions raises the question of whether decisions regarding everyday functions are also compromised by reduced memory awareness. Indeed, individuals with MCI and dementia are often unaware not only of cognitive deficits but of functional limitations as well $[1,8-11]$. Such unawareness may explain why a patient who needs assistance with daily tasks might make a less than optimal decision as to how to best carry out the task. For example, reduced awareness of cognitive and functional changes in patients with dementia can result in unsafe behaviors such as continued driving [12].

The current study sought to examine how awareness influences everyday decision making capacity related to medication management, a critical daily responsibility for many older adults. Medication management and adherence can be challenged by cognitive and metacognitive limitations in the context of dementia, and lack of illness awareness has been raised as a particular threat that should alert clinicians to potentially poor adherence [13]. We cross-sectionally examined the effects of memory awareness on decision making capacity related to medication management in early AD. We proposed that individuals with reduced awareness would make suboptimal decisions about medication management (e.g., failure to use assistive devices or enlist help from family members). Specifically, we hypothesized that reduced awareness would be associated with lower capacity scores secondary to impaired scores on the appreciation and reasoning indices.

What is especially novel about this study is that we investigated the influence of awareness on everyday decision making, using both subjective and objective metrics to comprehensively characterize memory awareness. We have shown that objective metamemory tools capture the clinical phenomenon of disordered awareness in $\mathrm{AD}[14]$ and have the potential to advance our understanding of its etiology, nature, and consequences [15]. While related, however, clinical ratings of awareness and metamemory scores also have the potential to capture different aspects of selfassessment. For example, while a subjective clinical rating of awareness characterizes an individual's broad perception of their memory, the objective metamemory task measures individuals' ability to systematically evaluate themselves on an item-by-item basis in the context of a specific task. Investigation of both awareness variables in relation to decision making may further elucidate the manner in which self awareness may affect capacity. Moreover, examining the precise factors which contribute to decision making will better equip clinicians and researchers to identify individuals with impairments in their capacity for navigating important decisions on a daily basis.

\section{Methods}

2.1. Participants. Given the cognitive demands of the metamemory task and our interest in studying capacity in early $\mathrm{AD}$, only patients with mild-to-moderate $\mathrm{AD}$, defined as a score of 19 or greater on the Mini-Mental State Examination (MMSE) [16] were recruited at two separate centers, Columbia University Medical Center and the University of Pennsylvania. Excluded were individuals with ongoing moderate-to-severe psychiatric conditions, and individuals with history of head injury, stroke, and other neurologic illnesses that might impact cognition and/or the presentation of $\mathrm{AD}$.

Columbia University Medical Center (CUMC). 17 individuals with mild $\mathrm{AD}$ were recruited through the CUMC Alzheimer's Disease Research Center and received comprehensive neurologic and neuropsychological evaluations that were reviewed in a diagnostic consensus conference attended by neurologists and neuropsychologists. Diagnoses of Alzheimer's disease were made according to the neurologic disorders and stroke-Alzheimer's disease and related disorders association (NINDS-ADRDA) criteria. All participants provided informed consent and were reimbursed $\$ 30.00$ for participation.

50 healthy elders were recruited from three sources: the healthy control database available through the Alzheimer's Disease Research Center at CUMC, local senior centers, and market mailing procedures that target a diverse group of elders in New York City with a range of ethnic and educational backgrounds. Controls were thoroughly screened by interview to exclude individuals with neurologic, psychiatric, or severe medical disorders. Participants were considered eligible for the study if they were age 55 or above, and scored at least 24 on the MMSE.

University of Pennsylvania. 25 individuals with mild $\mathrm{AD}$ were recruited through the University of Pennsylvania PENN Memory Center. Eligible patients and their family members enrolled at the Center, who agreed to be contacted for research studies, were sent a letter describing the study. A research assistant then called the contact person, and explained the study in more detail.

2.2. Procedures. Participants were seen for a two-hour test session including a clinical rating of awareness, capacity interview, metamemory testing, depression questionnaire, and a test of global cognition. Individuals seen at CUMC also underwent verbal episodic memory testing. Clinical ratings of awareness were not obtained on healthy elders as clinical diagnosis of $\mathrm{AD}$ was the criteria against which self-report was measured. For the purposes of the capacity interview, informants were contacted prior to the test session to obtain the relevant information. Informants were not required for healthy elders. This study was approved by the Institutional Review Board at both medical centers and all individuals provided informed consent prior to participation.

\subsection{Measures}

\subsubsection{Global Cognition}

Mini-Mental State Examination [16]. This commonly used 30-item measure of global cognition assesses orientation, attention, language, visuospatial functioning, and memory. 


\subsubsection{Memory}

Philadelphia Repeatable Verbal Learning Test (PVLT). [17] The PVLT is a list-learning task modeled after the 9-word California Verbal Learning Test $[18,19]$ in which participants are required to learn 9 words (comprising three different categories: fruit, tools, and furniture) over the course of five trials. The primary dependent variable was the recognition discriminability index, a variable that has been shown to reflect hippocampal integrity and to be differentially affected in $\mathrm{AD}$ versus other dementias [20].

\subsubsection{Decision-Making Capacity}

Assessment of Capacity for Everyday Decision Making (ACED) [21]. This semistructured interview consists of 30 items spanning the four abilities that constitute decision making capacity: understanding, appreciation, reasoning, and expression of choice. This assessment tool was designed to evaluate a person's capacity to solve problems with each of three daily decisions related to medication management, meal preparation, or finances. In the current study, analyses of decision-making capacity were restricted to those individuals who completed the medication management interview, as only 5 individuals completed the meal preparation and finances interviews.

The interview takes approximately 20 minutes to administer and is based on a discussion with a knowledgeable informant who confirms whether the participant has difficulty managing medications and what the current procedure is for managing the patient's medications. (Copies of the instrument can be obtained from Dr. Jason Karlawish.) Briefly, the Understanding subscale (0-10) assesses the individual's ability to comprehend information about options for medication management for "individuals who have memory problems" (e.g., use of a pillbox or assistance from another person), the advantages of these options, and the disadvantages. To reduce demands on memory, this information is presented orally to the subject and also printed on a sheet; the interviewer reminds the subjects that this information is available if they have forgotten what was presented orally by the examiner. In order to receive credit for answers, subjects must respond in their own words and not read verbatim from the sheet. The appreciation subscale (0-8) assesses individuals' ability to apply the information to themselves by asking them if they have any problems with remembering to take their medication and whether they think one of the suggested approaches to managing medications would benefit them or perhaps make things harder for them. The reasoning subscale (0-10) requires subjects to evaluate options for medication management in comparison to one another and to imagine what the effects of each option would be on their daily life. Finally, expression of choice (0-2) requires subjects to state a preference for managing their medications that is consistent with their reasoning throughout the interview.

Every interview was audio recorded for scoring and reliability purposes. All items were scored on a scale of 0 2. All interviews with participants with $\mathrm{AD}$ were scored by group consensus, and one out of every three interviews with healthy elders was consensus scored. At CUMC, the consensus group included at least two research assistants and the PI (SC). At HUP, the consensus group included two research assistants. A random sample of interviews obtained at CUMC were reviewed and scored by the team at Penn to ensure reliability across sites.

\subsubsection{Awareness Measures}

Clinical Ratings of Awareness. Testing sessions began with a brief interview to allow the examiner to make a clinical judgment regarding participant awareness of memory loss in participants with AD. Examiners asked participants to discuss their opinions of their memory abilities at the current time and assigned a score ranging from 1 to 4 on a modified version of the Anosognosia rating scale [22]. Participants were scored according to the following four point ordinal rating system: 1 = full awareness (spontaneous complaint or ready admission of memory loss along with the recognition that the loss is consequential and abnormal. Loss related to dementia or neurologic disease); 2 = moderate awareness (spontaneous admission of memory loss; however, loss is discussed in the context of "normal" age related changes. No discussion of diagnosis); $3=$ shallow awareness (inconsistent or transient recognition of memory loss, or uncertainty regarding memory loss. Patients may acknowledge inconsequential memory loss); $4=$ no awareness (matter-of-fact denial of impairment in response to direct questions regarding memory loss). If spontaneous responses did not clearly fit into a specific rating category (e.g., "My memory is bad"), the examiner queried as appropriate (e.g., "Do you have a sense of why your memory is bad?") to extract sufficient information for assigning a score of 1-4. Responses were recorded verbatim and scored before moving onto the remainder of the battery.

\subsection{Metamemory Test}

Task Development. The current metamemory test, a modified episodic feeling of knowing task [14], was designed as part of a larger ongoing study on metamemory in $\mathrm{AD}$ and thus has two characteristics that require attention. First, all healthy elders were randomly assigned to one of three task conditions (described below). Therefore, in the current study, analyses were conducted to ensure comparable metamemory performance across all conditions before collapsing scores across healthy elders. Second, metamemory stimuli were slightly modified several times over the course of the study to improve the psychometric properties of the task. Therefore, analyses were conducted to ensure comparable metamemory performance across stimuli sets in both the healthy elder and $\mathrm{AD}$ groups.

Task Instructions and Format. The examiner read the following instructions, "During this task, I am going to tell you about five people. I will tell you their name and something about their background. Your task is to try to remember this information as best you can. Please listen carefully". Immediately after the first learning trial (e.g., Haxby wrote 
TABle 1: Demographics, cognition, and metacognition.

\begin{tabular}{|c|c|c|c|c|c|c|}
\hline & $\mathrm{HE}(n=50)$ & $\operatorname{UAD}(n=19)$ & $\operatorname{AAD}(n=23)$ & $F$ & $d f$ & $P$ \\
\hline Age & $68.00(7.40)$ & $78.52(6.51)$ & $77.26(9.24)$ & 0.27 & 1.40 & 0.608 \\
\hline Education & $15.66(2.30)$ & $14.83(2.76)$ & $16.79(3.24)$ & 4.50 & 1. 40 & 0.040 \\
\hline$\%$ Female & 58 & 48 & 47 & - & 1 & 0.610 \\
\hline$\%$ Caucasian & 78 & 83 & 100 & - & 1 & 0.079 \\
\hline MMSE (0 to 30$)$ & $29.24(1.27)$ & $24.35(2.84)$ & $24.37(3.02)$ & 0.01 & 1. 40 & 0.982 \\
\hline Memory & $0.94(0.09)$ & $0.70(0.17)$ & $0.68(0.15)$ & 0.08 & 1.15 & 0.779 \\
\hline Metamemory & $0.61(0.57)$ & $0.18(0.72)$ & $0.68(0.33)$ & 6.67 & 1.33 & 0.015 \\
\hline Depression & $1.60(2.00)$ & $2.88(3.60)$ & $3.00(2.39)$ & 0.01 & 1.29 & 0.917 \\
\hline
\end{tabular}

Note. $\mathrm{HE}=$ healthy elder; $\mathrm{UAD}=$ unaware group; $\mathrm{AAD}=$ aware group; $\mathrm{MMSE}=$ mini-mental state examination; Memory = recognition discriminability index; Mood $=15$ item GDS. Awareness groups were determined on the basis of the clinical rating scale ( $\mathrm{AAD}=$ fully-moderately aware; UAD $=$ shallowno awareness). Analyses presented for UAD versus AAD.

a nonfiction book about space travel; Corbett was a former mayor in Nevada, etc.), predictions for memory performance were acquired one at a time for each item by providing written questions on $8.5^{\prime \prime} \times 11^{\prime \prime}$ paper (e.g., Who was a former mayor of Nevada?) and the following prompt read aloud by the examiner: "There are eight possible answers on the next page. Will you know which one is right-Yes, Maybe, or No?" Once predictions were recorded, participants were provided with eight answer choices and asked to select the correct answer. The answer choices included the correct response, the correct answers for the remaining 4 stimuli (to control for basic familiarity effects), and 3 new distractors. In the standard condition, the tester then moved onto the next item. All participants with $\mathrm{AD}$ and approximately one third of healthy elders completed the standard condition; the remaining two thirds of the healthy elders completed the query and feedback conditions. In the query condition, participants were asked whether they thought their answer was correct (postdiction) prior to moving onto the next item. In the feedback condition, the examiner told the participant if their answer was correct or incorrect prior to moving onto the next item. This process was repeated for learning trials 24 resulting in a total of 20 metamemory judgments. Stimuli were presented in the same order across each of the four learning trials; questions and answer choices were presented in a pseudorandom order.

Dependent Variable. Resolution, or the relative accuracy of self-judgments, reflects the extent to which accuracy is high when predictions for performance are high, and accuracy is low when predictions are low. The nonparametric Goodman-Kruskal gamma statistic, a rank order correlation, [23] was used to measure resolution. Gamma compares the relative number of concordant and discordant prediction/accuracy pairs, discarding "ties", or instances in which either the rating or accuracy in one pair is equal to that in another pair. Limitations of gamma include a tendency to be pulled to an extreme value on the basis of only one concordance or discordance and a possibility that no score can be calculated in the event of all ties. Although there are many potential metamemory metrics, gamma was used in the current study based on its selective association with clinical ratings of memory awareness in $\mathrm{AD}$ in a previous study from our lab [14], and its robustness as a measure of self-specific processes in nondemented elders [24].

\subsubsection{Mood}

15-Item Geriatric Depression Scale (GDS). The GDS measures a variety of depressive symptoms (e.g., sadness, hopelessness, and worthlessness) and has demonstrated adequate validity against the 30-item questionnaire [25] as well as concurrent validity with other measures of depression [26].

\section{Results}

The following results were considered significant at $P<.05$.

Clinical Ratings of Awareness. 19\% $(n=8)$ of AD participants were rated as fully aware of their memory loss, $26 \%$ $(n=11)$ were rated as moderately aware, $38 \%(n=16)$ were rated as having shallow awareness, and $17 \%(n=7)$ were judged to have no awareness of memory loss. $45 \%$ of the sample (full-moderate awareness) was collapsed into the Aware AD (AAD) group, and 55\% of participants (shallowno awareness) were compiled into the unaware AD (UAD) group. Consistent with our previous report, the awareness groups were indistinguishable on the basis of age, gender, global cognition, or memory. See Table 1. However, awareness groups in the current study differed slightly in years of education, with the aware group having approximately two more years of formal schooling $(P=.04)$. There was not a statistically significant difference in the proportion of aware $(29 \%)$ versus unaware $(10 \%)$ individuals who managed their medication independently $\left(x^{2}=1.954, P=.16\right)$.

Metamemory Task Condition and Stimuli Set. As part of a larger study, healthy elders received one of three conditions of the metamemory test. Of those who had a calculable metamemory score in the current study (35 of 50 healthy elders), $31 \%$ received the standard task condition, $40 \%$ the query, and 29\% the feedback. There was no difference in metamemory score as a function of task condition, $F(2,32)=1.63, P=.213$. There was also no difference in 
TABLE 2: Components of decision making for medication management.

\begin{tabular}{|c|c|c|c|c|c|c|}
\hline & $\mathrm{HE}$ & UAD & $\mathrm{AAD}$ & $F$ & $d f$ & $P$ \\
\hline ACED total & $28.94(1.85)$ & $22.33(6.13)$ & $25.56(3.24)$ & 3.64 & 1.35 & .065 \\
\hline Understanding & $9.48(0.93)$ & $7.52(2.34)$ & $7.87(2.06)$ & 0.23 & 1.35 & .637 \\
\hline Appreciation & $7.80(0.57)$ & $5.43(2.25)$ & $7.19(1.05)$ & 8.36 & 1.35 & .007 \\
\hline Comparative reasoning & $3.80(0.57)$ & $2.95(1.43)$ & $3.69(0.60)$ & 3.70 & 1.35 & .062 \\
\hline Consequential reasoning & $3.86(0.41)$ & $2.43(1.43)$ & $3.00(1.26)$ & 1.59 & 1.35 & .215 \\
\hline Expression of choice & $4.00(0.00)$ & $4.00(0.00)$ & $3.81(0.54)$ & 2.52 & 1.35 & .122 \\
\hline
\end{tabular}

Note. $\mathrm{HE}=$ healthy elder; $\mathrm{UAD}$ = unaware group; $\mathrm{AAD}$ = aware group; $\mathrm{ACED}=$ assessment of capacity for everyday decision making. Analyses presented for $\mathrm{UAD}$ versus AAD.

metamemory as a function of stimuli set in the healthy elders $F(2,32)=.10, P=.905$, or $\mathrm{AD}$ group $F(1,33)=.68$, $P=.415$. Therefore, all metamemory scores were collapsed across task condition and stimuli set for the current analyses.

Metamemory Scores. Gamma scores were calculable for 35 of 42 participants in the $\mathrm{AD}$ group ( $83 \%)$ and 35 of 50 healthy elders $(70 \%)$. The remaining participants demonstrated no variability in their predictions (yes, maybe, and no) or in their accuracy. In a replication of our previous findings, gamma scores in the $\mathrm{AD}$ group were significantly lower in the unaware group than in the aware group $(P=.015)$. See Table 1 for results by awareness group in individuals with $\mathrm{AD}$.

Decision Making Capacity. In the $\mathrm{AD}$ group, analyses examining capacity were restricted to the 34 individuals who completed the medication management version of the ACED. Mean scores for the ACED total score and subscores by awareness group are presented in Table 2 . A multivariate general linear model was used to compare ACED scores across the two AD groups and healthy elders. Analyses revealed that ACED scores were significantly lower in each of the $\mathrm{AD}$ groups than in the healthy elders $(F(2,67)=$ 17.63, UAD, $P<.01$; AAD, $P=.01)$. Performance on the ACED did not vary as a function of whether or not participants managed their medications independently at home $F(1,32)=.011, P=.92$.

Memory Awareness and Capacity. A univariate analysis of variance was used to examine capacity scores as a function of clinically determined awareness level in the $\mathrm{AD}$ group. Results are reported in Table 2. Total ACED score was not significantly different across groups $(P=.065)$, but performance on the appreciation index was lower in the UAD group as compared to the AAD group $(P=.007)$. This difference was driven both by problems appreciating one's own difficulty with managing medications $F(1,32)=5.596$, $P=.024$, as well as the overall ability to appreciate the potential benefits and harms of receiving assistance with medication management, $F(1,32)=4.34, P=.045$. When examined individually, neither appreciation of potential benefits nor appreciation of harms were significantly different as a function of awareness.

Bivariate correlations were then used to examine the correlates of decision making in $\mathrm{AD}$. Results are reported in
TABLE 3: Correlates of everyday decision making (ACED total score) in $\mathrm{AD}$.

\begin{tabular}{lccc}
\hline & $r$ & $P$ & $n$ \\
\hline Age & -.08 & .637 & 37 \\
Education & .10 & .555 & 37 \\
MMSE & .31 & .066 & 37 \\
Memory & -.02 & .940 & 16 \\
Clinical rating of awareness & -.41 & .007 & 42 \\
Metamemory score & -.10 & .599 & 31 \\
Depression & -.22 & .917 & 37 \\
\hline
\end{tabular}

Note. MMSE $=$ mini-mental state examination; Memory $=$ recognition discriminability index; Depression $=15$-item GDS.

Table 3. Clinical rating of awareness was the only characteristic that correlated with everyday decision making in $\mathrm{AD}$ $(r=-.41, P=.007)$.

\section{Discussion}

The current study investigated the selective influence of memory awareness in Alzheimer's disease on everyday decision-making capacity related to medication management. As predicted, and consistent with Karlawish and colleagues' findings in a study of decisions regarding memory treatment [5], awareness was particularly important for decision making capacity in the current study. That is, in comparison to demographic variables, global cognition, memory, and mood, only memory awareness was associated with everyday decision making. In particular, memory awareness was clearly associated with performance on the ability to appreciate personal difficulty with managing medications, as well as the advantages and disadvantages of receiving assistance with medication management. These results suggest that reduced memory awareness in $\mathrm{AD}$ may pose a particularly important threat to decision making, not only for medical treatment and research, but for everyday issues as well.

One of the advantages of the current study was the multiple metrics used to characterize memory awareness. In addition to rating participants from an overall, global, and clinical perspective, objective metamemory testing was used to quantify memory awareness rigorously and at a more local level. Previous work by our lab has established that scores on 
this metamemory test track with clinical ratings of awareness [14], and application of both methodological approaches increases our confidence that we are capturing the phenomenon of disordered awareness in a reliable fashion. Moreover, it enables consideration of the specific awareness components which may or may not influence the outcome of interest, in this case, everyday decision-making capacity.

Findings from the current study reinforce the idea that in the context of early $\mathrm{AD}$, awareness varies significantly, and this variation is not simply a reflection of global cognition, demographic variables, mood, or memory. Instead, it is a selective deficit. Its clinical consequences are important and relate to caregiver burden $[27,28]$, the success of behavioral interventions [29], decisions to continue driving [12], and medical decision making [5]. It is thus reasonable to expect that memory awareness may also influence everyday decisions that should be based in part on the fact that one's memory is impaired. Examination of medication management decisions allowed us to answer this question, given the idea that optimal decisions about medication management would require appreciation of one's memory limitations and the need to accommodate one's approach to this cognitively demanding daily routine in light of these limitations.

Examination of capacity scores across awareness groups in $\mathrm{AD}$ indeed revealed that decision making-capacity related to medication management was diminished in the unaware group, specifically on the appreciation subscale. The appreciation subscale queries the subjects to declare if they (1) have any problems with remembering to take medications, (2) think that using an assistive device (e.g., pillbox) to help them remember to take their medications could benefit them, (3) think that having someone administer their medications to them could benefit them, and (4) think that either of these options might actually make things worse for them.

These answers are always interpreted and scored in the context of the information provided by the informant. That is, if a subject denied a problem with remembering to take medications and this was contradicted by the informant, then a score of zero would be assigned for the first appreciation item. The remaining appreciation items required subjects to consider whether or not receiving assistance with medication management (in the form of a device such as a pillbox or help from another individual) could possibly benefit them or perhaps make things worse for them, and to justify their responses with a logical reason. Points were deducted if the response reflected false beliefs (e.g., I would not get any benefit from a pillbox because I do not have any trouble managing my medications) or illogical reasoning. In contrast, the understanding subscale does not require the individual to consider the decision from a personal standpoint, but simply to reiterate the options for someone who might need assistance with medication management, to consider the benefits in general, and the disadvantages in general. It is thus apparent why appreciation scores would be most affected by awareness: subjects must recognize their own difficulty with managing their medications. Interestingly, results indicate that unaware subjects not only had difficulty recognizing their problems with medication management (which might be expected), but they also had difficulty appreciating the potential advantages and disadvantages of receiving assistance.

The current findings nicely demonstrate that awareness selectively affects a component of capacity that requires application of information to oneself and not those indices which at face value have little to no metacognitive demands. We should emphasize that we are not arguing that primary cognitive functions are not important for capacity; of course, they contribute to performance across the various capacity subscales. Indeed, in the current study, both the aware and unaware $\mathrm{AD}$ groups had reduced capacity scores in relation to healthy elders, and studies consistently demonstrate associations between capacity and aspects of neuropsychological functioning in different clinical populations (see [30] for a review). What is interesting about the findings in the current study is that in the context of very-mild-to-mild $\mathrm{AD}$, the primary factor driving capacity was self-awareness rather than global cognition. Moreover, this was not simply a reflection of the task's hypothetical component (which could be cognitively demanding) as actual medication management status, that is, whether or not the participant received assistance with medication management at home, was comparable across awareness groups and unrelated to ACED scores. We cannot exclude the possibility that a specific cognitive deficit such as executive dysfunction mediated the association between capacity and awareness, as both variables have been associated with this cognitive deficit [31-33], and future work should address this possibility. However, a compelling study by Koren and colleagues demonstrating a selective role for metacognitive rather than executive abilities in predicting capacity to consent to treatment in individuals with schizophrenia speaks to this issue [34]. Specifically, on a modified version of the Wisconsin card sorting test (WCST) in which participants were asked to indicate whether or not they wanted each sort "counted" toward their overall score, multiple aspects of metacognition were related to capacity, whereas conventional WCST "executive" scores were unrelated. Moreover, recent work from our lab examining nondemented elders supports the idea that self-referential processing is independent from more general executive abilities [24]. In sum, it appears that aspects of metacognition are dissociable from more general executive abilities and may hold a particularly critical role for decision-making capacity.

This brings us to a second issue addressed in the current study, which was whether or not specific measures of memory awareness are differentially associated with capacity. Interestingly, despite the association between the two memory awareness measures in the current study (clinical ratings of awareness and metamemory scores), there was no association between metamemory scores and capacity (Table 3). This discrepancy touches on the differences between the two awareness metrics and urges us to consider what forms of memory awareness are captured uniquely by each measure despite their shared variance. We have argued in the past that objective metrics of memory awareness are critically needed for furthering our understanding of disordered awareness in $\mathrm{AD}$, particularly its nature and etiology [15], and have shown that such metrics in fact capture clinical differences in 
awareness [14]. Thus, use of objective metrics would be ideal when attempting to shed light on the specific metacognitive errors that give rise to the clinical phenomenon of disordered awareness, for example.

However, it may be that under certain circumstances, broad clinical impressions capture an element of self awareness that is practically relevant and lost when measured at the item level for a number of different reasons. Specifically, the metamemory task requires individuals to make itemby-item predictions about performance (i.e., local level) whereas the clinical rating of awareness queries for a much broader assessment of oneself (i.e., global level). Each type of self-assessment might affect the other such that general impressions about oneself might bias answers at the local level, and impairment at the local level might lead to impairment at the global level. However, global impressions of one's functioning may hold more weight for decision making than local assessments on a specified task, because the former represent beliefs about oneself in general rather than within the confines of a specific task. Such global beliefs are likely to be the basis on which we make decisions.

Moreover, an overall self-assessment that is outside the context of a task does not provide the opportunity for an individual to evaluate themselves online and incorporate any feedback about task performance; in that sense, it better approximates an individual's general sense of themselves and their abilities. Again, this type of self-awareness is what appears to be critical in the context of a decision-making paradigm in which individuals are required to consider a particular issue and to come to a decision about the issue in a context-free (task-free) environment. For example, the current capacity assessment was not conducted by requiring individuals to engage in a task (e.g., fill prescriptions, organize pills in a pillbox and prospectively remember to take these pills) before making a decision about how best to approach this task in daily life. Perhaps this more specific form of capacity assessment would be more highly related to awareness as measured through metacognitive testing.

This raises an interesting question about the manner in which decision-making capacity is assessed and might be assessed in the future. For example, some capacity instruments like the one used in this study intentionally reduce demands on episodic memory when evaluating capacity in patients with AD. Thus, patients are not penalized for this particular cognitive limitation, and other elements of decision making can be examined more directly. Similarly, perhaps heightening subjects' awareness in the context of a capacity evaluation would allow examination of decision making when an individual is provided with all information relevant to the decision. This might be achieved in a number of ways including (1) making explicit statements (e.g., "You have been diagnosed with Alzheimer's disease and this disease causes your memory functioning to decline over time"), (2) providing a context in which subjects might recognize memory failures (e.g., cognitive testing), or (3) conducting metacognitive testing to inform the patient about his or her memory awareness. While there may be ethical issues to consider for each of these approaches, the advantage would be that decision making could be evaluated once subjects possess accurate information about their abilities and would thus not be biased in subjects who have metacognitive deficits.

Alternatively, it may be that assessments of capacity are best when they approximate the "real world." If so, then an effort to facilitate awareness in the artificial context of a capacity evaluation would provide an inaccurate depiction of an individual's decision-making capacity in everyday life. For example, Marson and colleagues do not provide materials that reduce memory demands in their capacity assessment tools, and in fact, memory becomes a highly predictive factor for performance in individuals with dementia $[35,36]$. This may be a better representation of certain decisionmaking situations encountered by individuals in daily life, and thus an important part of the capacity evaluation. There is not likely to be one correct approach, and the specific circumstances of each capacity question and evaluation may have to drive the manner in which capacity is assessed, with the goal of balancing the patient's autonomy and safety as best as possible. Future work should further consider these issues and explore the feasibility and utility of different forms of capacity assessment in individuals with dementia.

In sum, results from the current study identify memory awareness as an important component of everyday decision making in $\mathrm{AD}$ and shed light on aspects of capacity assessments that may warrant additional consideration depending on the particular issue at hand. There are several limitations to the current study. First, it should be emphasized that overall judgments of capacity were not made in a dichotomous fashion by expert raters as has been done in other studies. As such, the current results do not comment on whether awareness directly affects the rating of an individual as capable or not capable to make daily decisions about medication management. Rather, current results allow the examination of the factors which contribute to decision making capacity, and which might be expected to lead to the judgment of an individual as not capable of making a decision. Second, memory data were unavailable for the individuals seen at the University of Pennsylvania; however, the correlation coefficient $(r=-.10)$ reported based on the individuals at CUMC is not suggestive of even a borderline statistical association between memory and capacity. Moreover, the manner in which capacity was measured in the current study was specifically selected to eliminate the demands on episodic memory, so an association was not anticipated. Third, the cognitive battery used in the current study was extremely limited as this was not the focus of the current investigation. However, future work should examine the extent to which specific aspects of cognition covary with awareness and capacity and whether or not deficits in any area of cognition might mediate the association between capacity and awareness. Finally, the current study did not include an objective measure of medication management $[37,38]$, a component which would have allowed us to directly examine the association between capacity, awareness, and the skills necessary for medication management. Despite these limitations, the current study provides important information on the factors which influence everyday decision making in $\mathrm{AD}$, highlighting the critical role for memory 
awareness and raising considerations for the manner in which capacity is currently assessed.

\section{Acknowledgments}

The authors would like to thank Dr. Yaakov Stern for his guidance and contributions during the conceptualization of this project. They would also like to thank the individuals who participated in this study, as well as the research assistants involved in collecting and scoring the data including Kristin Harkins, Paige Brookstein, Jonathan Rubright, Liz Sullo, Brittany Holmes, Rebecca Winter, and Preeti Sundararamen. This work was supported by a Paul B. Beeson Career Development Award in Aging (Cosentino: 1 K23 AG032899-01). J. Metcalfe is supported by NIMH RO1MH60637 and DoE R305H030175. J. Karlawish is supported by NIA P30-AG10124 and the Marian S. Ware Alzheimer Program.

\section{References}

[1] D. C. Marson, R. C. Martin, V. Wadley et al., "Clinical interview assessment of financial capacity in older adults with mild cognitive impairment and Alzheimer's disease," Journal of the American Geriatrics Society, vol. 57, no. 5, pp. 806-814, 2009.

[2] M. P. Dymek, P. Atchison, L. Harrell, and D. C. Marson, "Competency to consent to medical treatment in cognitively impaired patients with Parkinson's disease," Neurology, vol. 56, no. 1, pp. 17-24, 2001.

[3] O. C. Okonkwo, H. R. Griffith, J. N. Copeland et al., "Medical decision-making capacity in mild cognitive impairment: a 3year longitudinal study," Neurology, vol. 71, no. 19, pp. 14741480, 2008.

[4] B. S. Black, J. Brandt, P. V. Rabins et al., "Predictors of providing informed consent or assent for research participation in assisted living residents," American Journal of Geriatric Psychiatry, vol. 16, no. 1, pp. 83-91, 2008.

[5] J. H. T. Karlawish, D. J. Casarett, B. D. James, and S. X. Xie, "The ability of persons with Alzheimer disease $(\mathrm{AD})$ to make a decision about taking an $\mathrm{AD}$ treatment," Neurology, vol. 64, no. 9, pp. 1514-1519, 2005.

[6] P. S. Appelbaum and T. Grisso, "Assessing patient's capacities to consent to treatment," The New England Journal of Medicine, vol. 319, no. 25, pp. 1635-1638, 1988.

[7] P. S. Appelbaum and L. H. Roth, "Competency to consent to research. A psychiatric overview," Archives of General Psychiatry, vol. 39, no. 8, pp. 951-958, 1982.

[8] B. H. DeBettignies, R. K. Mahurin, and F. J. Pirozzolo, "Insight for impairment in independent living skills in Alzheimer's disease and multi-infarct dementia," Journal of Clinical and Experimental Neuropsychology, vol. 12, no. 2, pp. 355-363, 1990.

[9] S. T. Farias, D. Mungas, and W. Jagust, "Degree of discrepancy between self and other-reported everyday functioning by cognitive status: dementia, mild cognitive impairment, and healthy elders," International Journal of Geriatric Psychiatry, vol. 20, no. 9, pp. 827-834, 2005.

[10] O. C. Okonkwo, H. R. Griffith, D. E. Vance, D. C. Marson, K. K. Ball, and V. G. Wadley, "Awareness of functional difficulties in mild cognitive impairment: a multidomain assessment approach," Journal of the American Geriatrics Society, vol. 57, no. 6, pp. 978-984, 2009.

[11] O. C. Okonkwo, V. G. Wadley, H. R. Griffith et al., "Awareness of deficits in financial abilities in patients with mild cognitive impairment: going beyond self-informant discrepancy," American Journal of Geriatric Psychiatry, vol. 16, no. 8, pp. 650-659, 2008.

[12] V. Cotrell and K. Wild, "Longitudinal study of self-imposed driving restrictions and deficit awareness in patients with Alzheimer disease," Alzheimer Disease and Associated Disorders, vol. 13, no. 3, pp. 151-156, 1999.

[13] S. Arlt, R. Lindner, A. Rösler, and W. von Renteln-Kruse, "Adherence to medication in patients with dementia: predictors and strategies for improvement," Drugs and Aging, vol. 25, no. 12, pp. 1033-1047, 2008.

[14] S. Cosentino, J. Metcalfe, B. Butterfield, and Y. Stern, "Objective metamemory testing captures awareness of deficit in Alzheimer's disease," Cortex, vol. 43, no. 7, pp. 1004-1019, 2007.

[15] S. Cosentiono and Y. Stern, "Metacognitive theory and assessment in dementia: do we recognize our areas of weakness?" Journal of the International Neuropsychological Society, vol. 11, no. 7, pp. 910-919, 2005.

[16] M. F. Folstein, S. E. Folstein, and P. R. McHugh, "'Mini mental state'. A practical method for grading the cognitive state of patients for the clinician," Journal of Psychiatric Research, vol. 12, no. 3, pp. 189-198, 1975.

[17] C. C. Price, K. D. Garrett, A. L. Jefferson et al., "Leukoaraiosis severity and list-learning in dementia," The Clinical Neuropsychologist, vol. 23, no. 6, pp. 944-961, 2009.

[18] D. Delis, J. H. Kramer, E. Kaplan, and B. A. Ober, California Verbal Learning Test: Adult Version Manual, The Psychological Corporation, San Antonio, Calif, USA, 1987.

[19] D. J. Libon, R. E. Mattson, G. Glosser et al., "A nine-word dementia version of the California verbal learning test," The Clinical Neuropsychologist, vol. 10, no. 3, pp. 237-244, 1996.

[20] D. J. Libon, B. Bogdanoff, B. S. Cloud et al., "Declarative and procedural learning, quantitative measures of the hippocampus, and subcortical white alterations in Alzheimer's disease and ischaemic vascular dementia," Journal of Clinical and Experimental Neuropsychology, vol. 20, no. 1, pp. 30-41, 1998.

[21] J. M. Lai, T. M. Gill, L. M. Cooney, E. H. Bradley, K. A. Hawkins, and J. H. Karlawish, "Everyday decision-making ability in older persons with cognitive impairment," American Journal of Geriatric Psychiatry, vol. 16, no. 8, pp. 693-696, 2008.

[22] B. R. Reed, W. J. Jagust, and L. Coulter, "Anosognosia in Alzheimer's disease: relationships to depression, cognitive function, and cerebral perfusion," Journal of Clinical and Experimental Neuropsychology, vol. 15, no. 2, pp. 231-244, 1993.

[23] T. O. Nelson, "A comparison of current measures of the accuracy of feeling-of-knowing predictions," Psychological Bulletin, vol. 95, no. 1, pp. 109-133, 1984.

[24] S. Cosentino, J. Metcalfe, B. Holmes, J. Steffener, and Y. Stern, "Finding the self in metacognitive evaluations: a study of metamemory and agency in non-demented elders," Neuropsychology. In press.

[25] E. L. Lesher and J. S. Berryhill, "Validation of the geriatric depression scale-short form among inpatients," Journal of Clinical Psychology, vol. 50, no. 2, pp. 256-260, 1994. 
[26] F. R. Ferraro and I. Chelminski, "Preliminary normative data on the Geriatric Depression Scale-Short Form (GDS-SF) in a young adult sample," Journal of Clinical Psychology, vol. 52, no. 4, pp. 443-447, 1996.

[27] B. Seltzer, J. J. Vasterling, J. Yoder, and K. A. Thompson, "Awareness of deficit in Alzheimer's disease: relation to caregiver burden," Gerontologist, vol. 37, no. 1, pp. 20-24, 1997.

[28] M. Spitznagel, J. Gunstad, J. O. Carvahlo, L. B. Brown, J. D. Davis, and G. Tremont, "Impaired awareness in dementia patients predicts caregiver burden," Journal of the International Neuropsychological Society, vol. 12, no. S1, p. 51, 2006.

[29] D. C. Koltai, K. A. Welsh-Bohmer, and D. E. Schmechel, "Influence of anosognosia on treatment outcome among dementia patients," Neuropsychological Rehabilitation, vol. 11, no. 3-4, pp. 455-475, 2001.

[30] B. W. Palmer and G. N. Savla, "The association of specific neuropsychological deficits with capacity to consent to research or treatment," Journal of the International Neuropsychological Society, vol. 13, no. 6, pp. 1047-1059, 2007.

[31] L. E. Dreer, M. J. DeVivo, T. A. Novack, S. Krzywanski, and D. C. Marson, "Cognitive predictors of medical decision-making capacity in traumatic brain injury," Rehabilitation Psychology, vol. 53, no. 4, pp. 486-497, 2008.

[32] D. C. Marson, H. A. Cody, K. K. Ingram, and L. E. Harrell, "Neuropsychologic predictors of competency in Alzheimer's disease using a rational reasons legal standard," Archives of Neurology, vol. 52, no. 10, pp. 955-959, 1995.

[33] A. Michon, B. Deweer, B. Pillon, Y. Agid, and B. Dubois, "Relation of anosognosia to frontal lobe dysfunction in Alzheimer's disease," Journal of Neurology Neurosurgery and Psychiatry, vol. 57, no. 7, pp. 805-809, 1994.

[34] D. Koren, M. Poyurovsky, L. J. Seidman, M. Goldsmith, S. Wenger, and E. M. Klein, "The neuropsychological basis of competence to consent in first-episode schizophrenia: a pilot metacognitive study," Biological Psychiatry, vol. 57, no. 6, pp. 609-616, 2005.

[35] K. S. Earnst, D. C. Marson, and L. E. Harrell, "Cognitive models of physicians' legal standard and personal judgments of competency in patients with Alzheimer's disease," Journal of the American Geriatrics Society, vol. 48, no. 8, pp. 919-927, 2000.

[36] M. G. Sherod, H. R. Griffith, J. Copeland et al., "Neurocognitive predictors of financial capacity across the dementia spectrum: normal aging, mild cognitive impairment, and Alzheimer's disease," Journal of the International Neuropsychological Society, vol. 15, no. 2, pp. 258-267, 2009.

[37] S. M. Albert, C. M. Weber, G. Todak et al., "An observed performance test of medication management ability in HIV: relation to neuropsychological status and medication adherence outcomes," AIDS and Behavior, vol. 3, no. 2, pp. 121-128, 1999.

[38] B. J. Gurland, P. Cross, J. Chen et al., "A new performance test of adaptive cognitive functioning: the Medication Management (MM) test," International Journal of Geriatric Psychiatry, vol. 9, no. 11, pp. 875-885, 1994. 


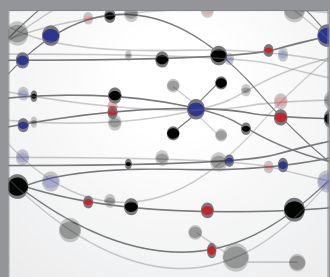

The Scientific World Journal
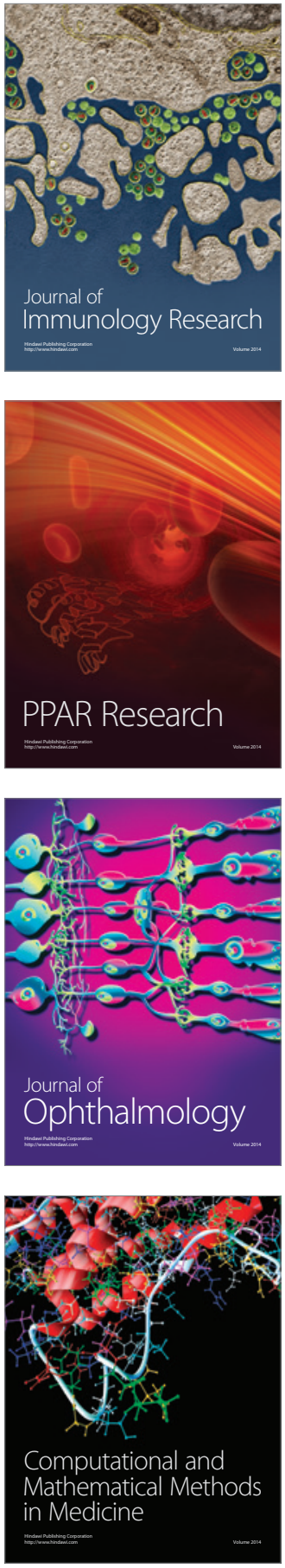

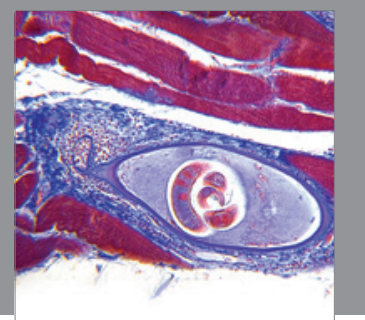

Gastroenterology

Research and Practice
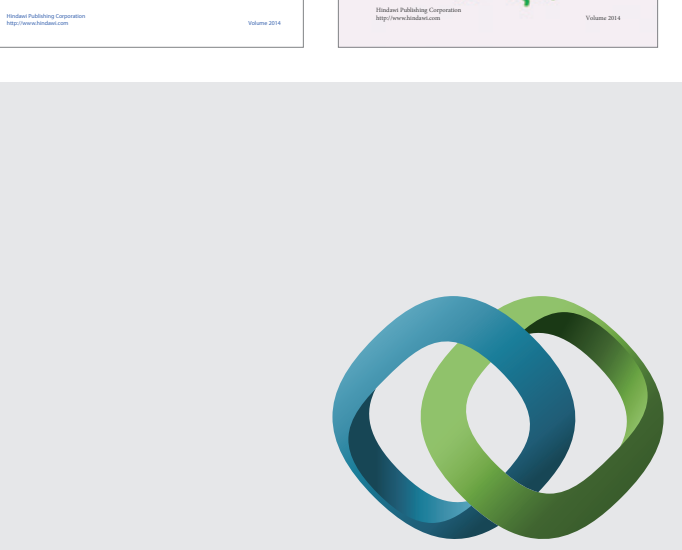

\section{Hindawi}

Submit your manuscripts at

http://www.hindawi.com
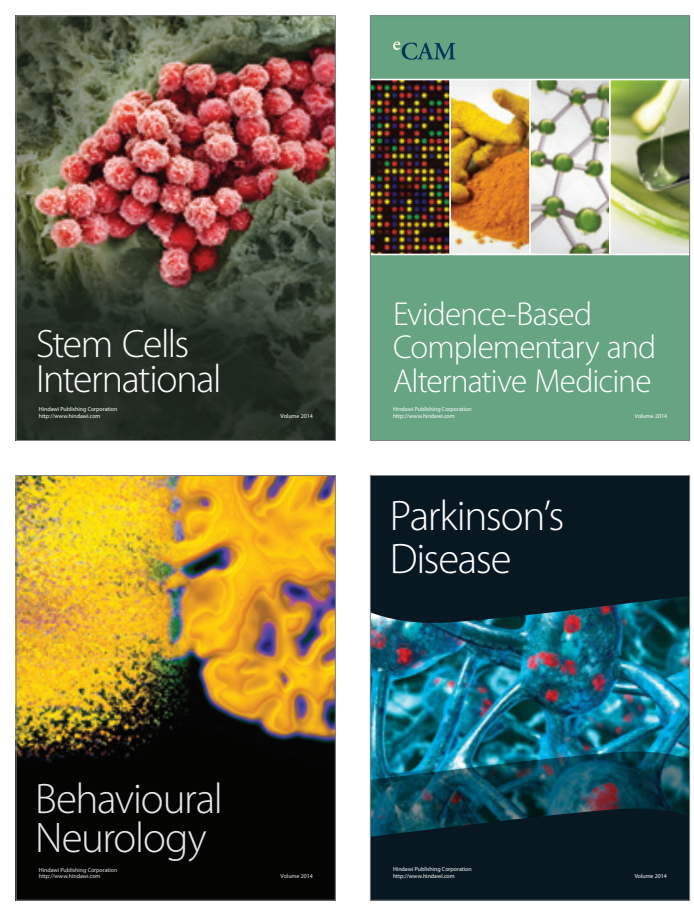

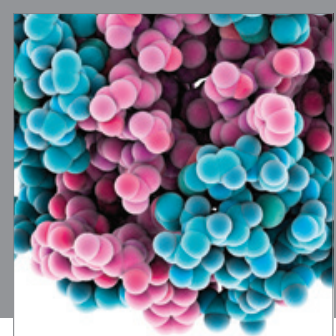

Journal of
Diabetes Research

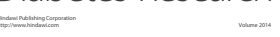

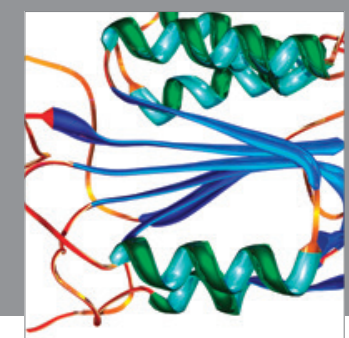

Disease Markers
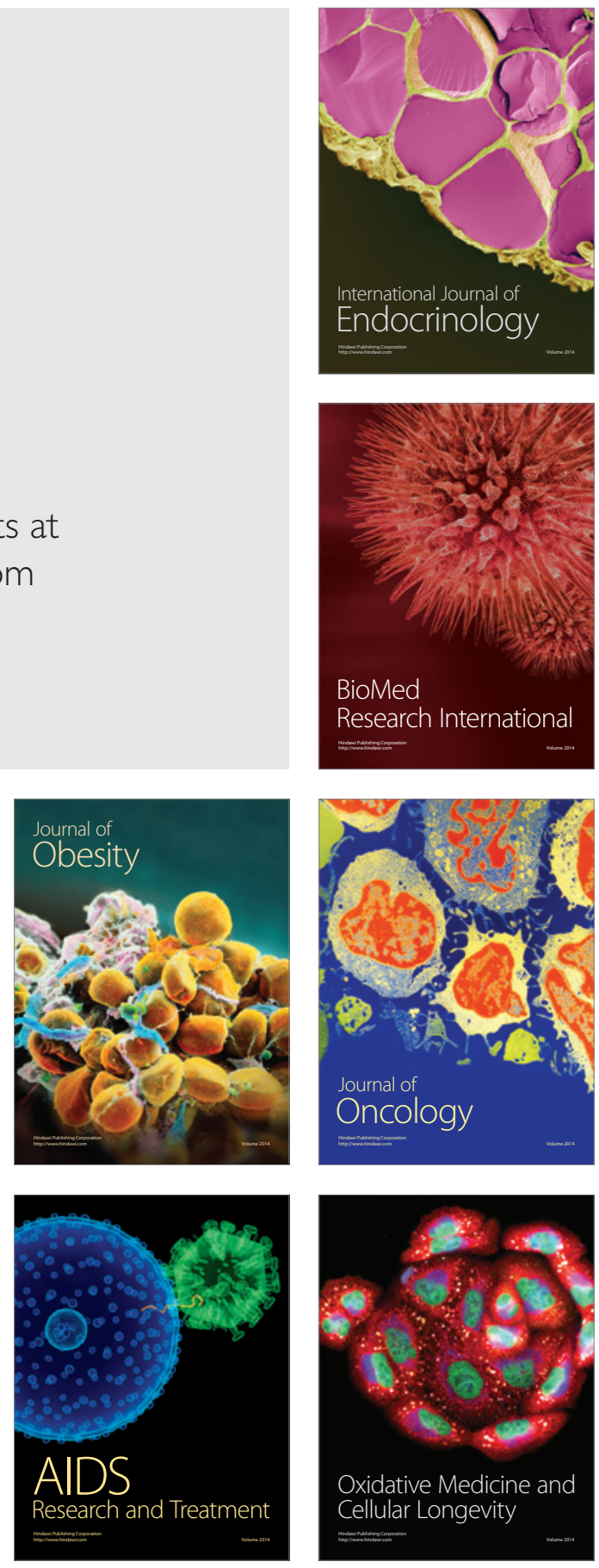